\title{
The contribution of patch topology and demographic parameters to population viability analysis predictions: the case of the European tree frog
}

\author{
Jérôme Pellet $\cdot$ Gérard Maze $\cdot$ Nicolas Perrin
}

Received: 10 September 2005 / Accepted: 16 May 2006

(C) The Society of Population Ecology and Springer-Verlag Tokyo 2006

\begin{abstract}
Population viability analyses (PVA) are increasingly used in metapopulation conservation plans. Two major types of models are commonly used to assess vulnerability and to rank management options: population-based stochastic simulation models (PSM such as RAMAS or VORTEX) and stochastic patch occupancy models (SPOM). While the first set of models relies on explicit intrapatch dynamics and interpatch dispersal to predict population levels in space and time, the latter is based on spatially explicit metapopulation theory where the probability of patch occupation is predicted given the patch area and isolation (patch topology). We applied both approaches to a European tree frog (Hyla arborea) metapopulation in western Switzerland in order to evaluate the concordances of both models and their applications to conservation. Although some quantitative discrepancies appeared in terms of network occupancy and equilibrium population size, the two approaches were largely concordant regarding the ranking of patch
\end{abstract}

\section{J. Pellet $\cdot$ N. Perrin}

Department of Ecology and Evolution,

Laboratory for Conservation Biology,

University of Lausanne, 1015 Lausanne, Switzerland

\section{J. Pellet ( $\square)$}

Department of Biological Sciences,

Center for Conservation Biology,

Stanford University, Stanford, CA 94305, USA

e-mail: jpellet@stanford.edu

\section{G. Maze}

Laboratory of Algorithmic Mathematics,

Mathematics Section,

Swiss Federal Institute of Technology,

Lausanne, Switzerland values and sensitivities to parameters, which is encouraging given the differences in the underlying paradigms and input data.

Keywords Population viability analyses - RAMAS Stochastic patch occupancy models - Extinction · Colonization - Dispersal distance $\cdot$ Hyla arborea. Patch occupancy $\cdot$ Population size $\cdot$ Switzerland

\section{Introduction}

Metapopulation modeling is being increasingly used for the development of wildlife conservation strategies (McCullough 1996; Akçakaya and Sjögren-Gulve 2000; Beissinger and McCullough 2002). The understanding of the mechanisms underlying extinction, dispersal and colonization in metapopulations is a key issue that needs to be addressed in order to estimate wild populations' vulnerability. To address this issue, two major types of population viability analyses (PVA) are being used: population-based stochastic simulation models (PSM) and stochastic patch occupancy models (SPOM) (Akçakaya and Sjögren-Gulve 2000).

Population viability analyses based on species-specific data have been intensively exploited in metapopulation frameworks during the last decade, mainly because user-friendly softwares were made available (e.g., VORTEX and RAMAS: Lindenmayer et al. 1995; Akçakaya 2002). Quantitative methods such as PSM present many advantages, including the ability to encapsulate all data and knowledge available for a given species and explicitly modeling intra- and interpatch dynamics. It also allows the identification, through sensitivity analysis, of specific demographic 
parameters on which conservation actions are most likely to be effective in order to prevent species extinctions. However, PSM suffer the drawback of requiring a large amount of population-level demographic data (e.g., fecundity and survival), information on population dynamics (e.g., stage structure, density dependence, dispersal, environmental and demographic stochasticity) in order to provide meaningful results. In PSM, the focus is mostly set on population and demographic processes rather than on habitat patches. Local populations can be structured in age classes, and demographic rates are assumed to be identical among all individuals of a given age class. Corresponding year-to-year transition matrices can be formulated in order to reflect the life history of the focal species, assuming discrete time steps. Environmental and demographic stochasticity is also implemented in those models (Akçakaya 2000, 2002). Such models aim at describing global population dynamics by modeling both local population dynamics and dispersal. This kind of PVA has been applied to a wide range of species, among which birds, butterflies and mammals share an important part (Akçakaya et al. 2004). Because in most cases estimates of vital rates and/or original population sizes were inaccurate, uncertain or lacunar, the application of such models was mostly done through sensitivity analyses in order to assess vulnerability, rank management options or plan data collection (Akçakaya and Sjögren-Gulve 2000).

Along with the development of spatially realistic metapopulation theory (Hanski 2001; Hanski and Ovaskainen 2003), SPOM emerged (Etienne et al. 2004). These models are also based on a spatially explicit patch network but do not, contrarily to PSM, explicitly describe local population dynamics. The SPOM have the ability to predict the equilibrium probability of a patch being occupied when given patch extinction and colonization probabilities. Contrarily to the PSM, this second set of metapopulation models, while requiring monitoring data on patch occupancy and/or turnover events, requires less information on the focal species vital rates, since it does not explicitly model intrapatch demography (Hanski 1994; SjögrenGulve 1994; Sjögren-Gulve and Ray 1996; Vos et al. 2000). Occupancy models such as SPOM aim at estimating the equilibrium proportion of patches occupied by a focal species; they do not keep track of local population dynamics (Sjögren-Gulve and Hanski 2000). They assume that patches are either occupied or empty, with local extinctions and colonization probabilities being dependent on the size and spatial configuration of all patches.
The first set of metapopulation models (PSM) is widely used in conservation planning (Kindvall 2000; Hels and Nachman 2002; Akçakaya et al. 2004; Schtickzelle and Baguette 2004), while the second set (SPOM) is somewhat restricted to a more theoretical field (although applied examples exist: Hanski and Ovaskainen 2000; Thomas et al. 2001; Moilanen and Cabeza 2002; Drechsler et al. 2003).

Although both approaches are being intensively discussed and criticized (Baguette 2004; Hanski 2004), only a few attempts have been made to evaluate their convergences and/or divergences in a real metapopulation (Kindvall 2000; Lopez and Pfister 2001) and their potential applications to conservation management. Here, we present the development of both a demographically explicit model (using RAMAS Metapop 3.0) and a SPOM for a metapopulation of the European tree frog (Hyla arborea L., 1758). It has been previously demonstrated that European tree frog populations undergo regular extinction and recolonization events (Carlson and Edenhamn 2000; Vos et al. 2000; Schmidt and Pellet 2005), fulfilling the metapopulation definition (Hanski and Simberloff 1997; Smith and Green 2005). Using demographic and occupancy data, we aimed at answering the following two questions: (1) Are the two approaches concordant regarding various indicators of metapopulation persistence in time? (2) Do sensitivity analyses of model input parameters identify a single relative importance for the same parameters? We finally discuss the implications of both approaches.

\section{Materials and methods}

Species and study area

Widely distributed across the Swiss Plateau at the beginning of the 20th century, the European tree frog has regressed to the point where only a few metapopulations now remain in the country (Grossenbacher 1988; Pellet et al. 2004). It is thus considered highly threatened in Switzerland (Schmidt and Zumbach 2005). Breeding and oviposition take place in gravel pits, military training grounds and other temporary wetlands (Grosse and Nöllert 1993). During the breeding period, males call conspicuously from pond shores to attract gravid females (Schneider 1993). Counts of calling males are thus used to evaluate the size of breeding populations (Carlson and Edenhamn 2000). The maximum number of calling males heard in a single visit each year is thought to reflect the annual breeding male population size (Edenhamn 1996; Pellet 
and Schmidt 2005). The remnant metapopulation that we modeled here has been described elsewhere (Pellet et al. 2004, 2005). It consists of 16 local populations (breeding ponds) located in a $225-\mathrm{km}^{2}$ area in western Switzerland, of which less than $0.5 \%$ consists of amphibian breeding ponds. Patch location is represented in Fig. 1. Patch carrying capacity is estimated as the mean maximum number of calling males heard during 4 years of survey (2001-2004).

Population-based stochastic simulation model (RAMAS)

We implemented a two-stage structured demographic model in RAMAS Metapop 3.0 in which only the sex that limits reproductive capacity (females) was included (assuming a 1:1 sex ratio). Population size assumes a pre-breeding census where the count of females is expected to equal the count of calling males. The first stage included all non-reproductive yearlings, while stage two is a complementary class including all reproductive individuals. Our model assumes that all individuals become reproductive on their 2 nd year (24 months after metamorphosis), based on skeletochronological studies of breeding aggregations (Friedl and Klump 1997). The corresponding year-to-year transition Leslie matrix is thus defined as:

$L=\left(\begin{array}{cc}0 & f_{\mathrm{a}} \\ s_{\mathrm{a}} & s_{\mathrm{a}}\end{array}\right)$,

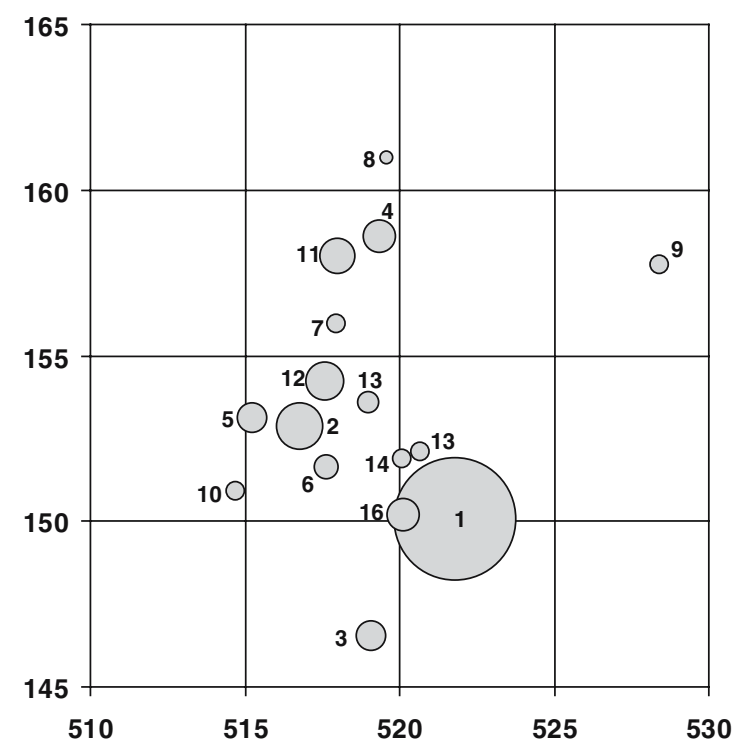

Fig. 1 Tree frog patch number and location. Patch surface is proportional to estimated carrying capacity. Scale is in kilometers based on the Swiss geographic reference system where $f_{\mathrm{a}}$ is the effective adult fecundity (which includes sex ratio, probability of egg laying, clutch size and all pre-yearling survival rates: Vonesh and De la Cruz 2002), and $s_{\mathrm{a}}$ is the yearly adult survival rate. The adult survival rate was estimated from a 4-year-long capturemark-recapture (CMR) experiment undertaken on three tree frog populations in Switzerland by Tester (1990). Eight year-to-year adult return rates (proportion of recaptured frogs) provide us with a minimum estimate (and standard deviation) of tree frog adult survival. This mean return rate was estimated to $s_{\mathrm{a}}=0.303(\mathrm{SD}=0.097)$. This value is consistent with our own observations in similar CMR experiments in western Switzerland (Pellet et al. 2006).

To evaluate $f_{\mathrm{a}}$, we used an indirect approach based on the relationship given by $\operatorname{det}(L-\lambda \mathrm{I})=0$ (Caswell 2001), which, assuming equilibrium population dynamics $(\lambda=1)$, allowed us to match fecundities to growth rate of 1 using the following formula:

$f_{\mathrm{a}}=\frac{1}{s_{\mathrm{a}}}-1$

The numerical resolution of (2) gave $f_{\mathrm{a}}=2.297$. All demographic rates were assumed to be affected by environmental stochasticity in a similar way (similar coefficient of variation $\mathrm{CV}$ ). The $\mathrm{CV}$ on $s_{\mathrm{a}}$ being estimated by $\mathrm{SD}\left(s_{\mathrm{a}}\right) / s_{\mathrm{a}}=32 \%$, we were able to estimate $\mathrm{SD}\left(f_{\mathrm{a}}\right)$ as $f_{\mathrm{a}} \cdot 32 \%=0.735$, leading to a full transition matrix $L$ :

$L_{s}=\left(\begin{array}{cc}0 & 2.297 \pm 0.735 \\ 0.303 \pm 0.097 & 0.303 \pm 0.097\end{array}\right)$

Demographic parameters at each time step of the simulations were randomly taken from lognormal distributions based on the above values. Given the above transition matrix $L$ (3), we simulated the dynamic of a single population over 50 years. We then compared the resulting $\operatorname{SD}(\lambda)$ with observed variations in the growth rate over 20 years in an isolated population near Lerchenfeld (Pellet et al. 2006). Standard deviation on the mean growth rate was estimated as $\mathrm{SD}\left(\lambda_{\text {RAMAS }}\right)=$ 0.860 , while the observed value was $\operatorname{SD}\left(\lambda_{\text {obs }}\right)=0.668$, thus suggesting that our transition matrix provided a reasonably good approximation of environmental stochasticity.

Evidence of density-dependent regulation has been highlighted in an analysis of long-term tree frog population dynamics (Pellet et al. 2006). We thus included a Ricker-type (linear or scramble) density dependence function for each population, assuming that density dependence affected both fecundity and 
survival. There is substantial evidence that density dependence can affect amphibians both in the pre- and post-metamorphic stages (Altwegg 2003). The maximum growth rate of all populations $\left(R_{\max }\right)$ was estimated as the intercept of the regression of growth rates on population sizes. Populations' carrying capacities $K_{i}$ were defined as the mean number of calling males heard in our set of 16 populations between 2001 and 2004 (assuming a stable age distribution).

The proportion of individual dispersing from patch $i$ to $j$ is defined as $m_{i j}=a \exp \left(-\alpha d_{i j}\right)$, where $a$ is the intercept value of the dispersal function (or dispersal rate), $1 / \alpha$ is the mean dispersal distance and $d_{i j}$ is the distance between patches $i$ and $j$. Data on European tree frog dispersal were provided by a large-scale experiment (Stumpel and Hanekamp 1986; Vos et al. 2000) that provided unbiased dispersal data (Smith and Green 2005). On a total of 89 dispersal events, they evidenced a mean dispersal distance of $1 / \alpha=1.469 \mathrm{~km}$ (maximum $12.570 \mathrm{~km}$ ). We set the yearlings' dispersal rate to $a=0.2$, and the adult dispersal rate was set to $10 \%$ of the yearlings' dispersal value (Sjögren-Gulve 1994).

The demographic model was applied to our set of 16 local populations in western Switzerland (Fig. 1) with initial population sizes set to values recorded in 2001 (counts of calling males, Pellet and Schmidt 2005). The yearlings' population was set assuming stable age structure given the transition matrix $L$. The baseline model included 10,000 simulations over 50 years. We modified this baseline model by changing the two density dependence parameters $R_{\max }$ and $K_{i}$ by $+10 \%$ and $-10 \%$, thus creating a new set of two models reflecting two population growth situations (see Akçakaya 2006).

Stochastic patch occupancy model

Our second modeling approach was derived from Hanski and Ovaskainen (2000) and Ovaskainen and Hanski (2001). Their model is a spatially realistic version of the Levins metapopulation model in which patch size and location contribute to the dynamics of the system. The rate of change in the probability of patch $i$ being occupied is:

$p_{i, t+1}=c_{i}\left(1-p_{i, t}\right)+\left(1-e_{i}\right) p_{i, t}$,

where $c_{i}$ and $e_{i}$ are patch-specific colonization and extinction rates defined as:

$c_{i}=c \sum_{i \neq j} e^{-\alpha d_{i j}} A_{j} p_{j}$

and $e_{i}=e \frac{1}{A_{i}^{\zeta}}$,

respectively. $A_{i}$ is patch $i$ size (or carrying capacity as defined previously), $d_{i j}$ is the distance between patches $i$ and $j$, and $1 / \alpha$ is the mean species dispersal distance. In the previous equations, $c$ and $e$ are species-specific colonization and extinction rates, $p_{j}$ is the probability of patch $j$ being occupied, and $\zeta$ is a scaling parameter defining the relationship between patch extinction rates $e_{i}$ and patch size $A_{i}$. The expected equilibrium patch occupancy may then be computed and metapopulation size may be extrapolated from both patch occupancy and the population carrying capacity.

Our application of this modeling approach used the same populations and carrying capacities as those used in the RAMAS model. Four additional parameters were needed to fully parameterize the model: $e, c, \alpha$ and $\zeta$. These values were extracted from a study of tree frog metapopulation dynamics undertaken by Carlson and Edenhamn (2000). During a 3-year study period, they estimated extinction rates as a function of patch size (defined as the count of calling males) in a set of 378 patches. Using their published data, we estimated $e$ and $\zeta$ from the intercept and slope of the following regression:

$\ln \left(e_{i}\right)=\ln (e)-\zeta \ln \left(A_{i}\right)$,

with $e_{i}$ being the observed extinction probability and $A_{i}$ being the local patch size. This approach led to $\zeta=0.388$ and $e=0.725$, with an explained variance of $87 \%$. The species dispersal ability was again set to $1 / \alpha=1.469 \mathrm{~km}$, as in the PSM. As the patch-specific colonization rate $c_{i}$ was not available from Carlson and Edenhamn (2000), we estimated $c$ by minimizing the discrepancies (the sum of squared residuals) between the expected (by the SPOM) and observed patch occupancy in the metapopulation during 2001-2004. This numerical approach provided $c=0.420\left(R^{2}=51 \%\right)$.

Average population size $N_{i}$ was computed as $N_{i}=A_{i} p_{i}$, where $A_{i}$ is patch $i$ carrying capacity and $p_{i}$ is the equilibrium probability of patch $i$ being occupied.

Model output comparison

Both models provide estimates of patch occupancy [mean patch occupancy (MPO) or the proportion of patches occupied] as well as the probability of any given patch being occupied. Similarly, they supply global metapopulation sizes in addition to the local patch population size mean final population size $(\mathrm{MFN})$. Because the extinction risk was too low in 
most simulations, it was not selected as a relevant model output. Concordance between our two baseline models regarding the probability of patch occupancy and mean local population size was evaluated using the Spearman ranked correlation coefficient.

\section{Sensitivity analysis}

Sensitivity analyses were run for both approaches. The MPO and MFN were used as sensitivity indicators (Conroy and Brook 2003). Input parameters were changed one at a time $( \pm 50 \%)$ from the baseline model, and resulting indicator values were reported. The direction of the parameter change was always chosen such as to reduce the metapopulation occupancy and final size. Parameters were ranked according to their impact on the final indicators, and a mean rank was computed in order to summarize information on input parameter sensitivity.

\section{Results}

Comparison of baseline models

Both baseline models reach the conclusion that equilibrium patch occupancy should be around 14-15 occupied patches with a total of 200-300 callers distributed in the whole metapopulation.

Comparison of baseline models of both approaches at the patch level provides good concordance for patch

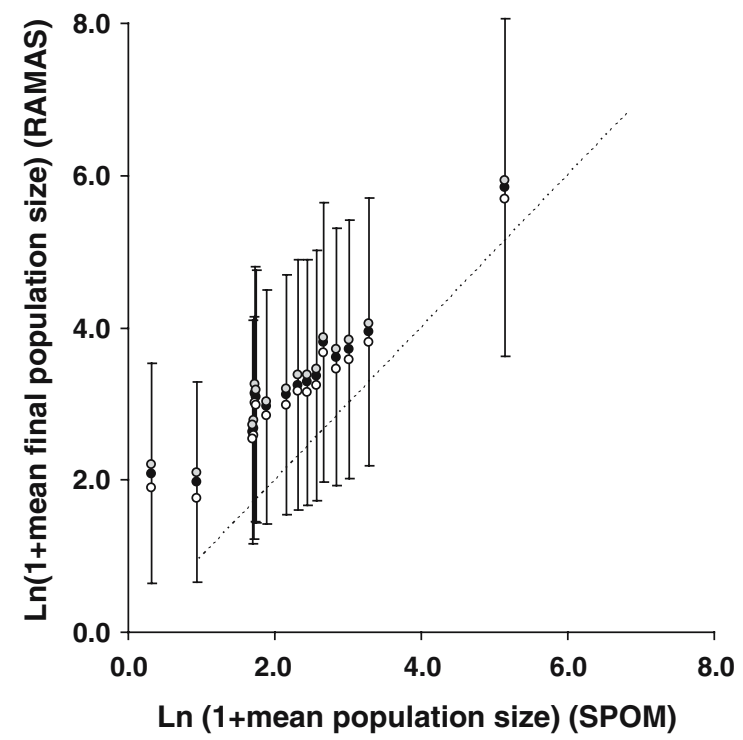

Fig. 2 Concordance between the two modeling approaches regarding probability of local patch occupation and mean final population size $( \pm \mathrm{SD})$. Variables were transformed for clarity. ranking, both in terms of the probability of patch occupancy and of population sizes (Fig. 2). Spearman's rank correlation between patches' MPO is good $\left(r_{\mathrm{s}}=0.810 ; P<0.001\right)$ and very good regarding patches' $\operatorname{MFN}\left(r_{\mathrm{s}}=0.968 ; P<0.001\right)$. There is, however, a quantitative deviation between the two models, the PSM having close to 1.5 times larger expected population size than the SPOM. Similarly, the PSM is optimistic compared to the SPOM, with occupancy rates on average 1.5 times larger. Taken together, these results indicate a good qualitative match, but some quantitative discrepancies between the results of the two modeling approaches.

\section{Sensitivity on the RAMAS model}

Sensitivity analysis (Table 1) showed that the maximum growth rate $R_{\max }$ has a preponderant effect on both MPO and MFN. The effect is, however, much stronger on the final metapopulation size $(-40 \%$ compared to the baseline model) than on patch occupancy $(-5 \%)$. With a mean third rank in our sensitivity analysis, the mean dispersal distance evidences the importance of dispersal on population size and persistence. The effect of this parameter is stronger on patch occupancy $(-8 \%)$ than on final metapopulation size $(-2 \%)$. Similarly, the other dispersal parameter (dispersal rate $a$ ) ranks on average third, but it appears that a relatively large modification in its baseline value $(-50 \%)$ modifies the final indicators MPO and MFN by less than $5 \%$. Standard deviation on demographic rates

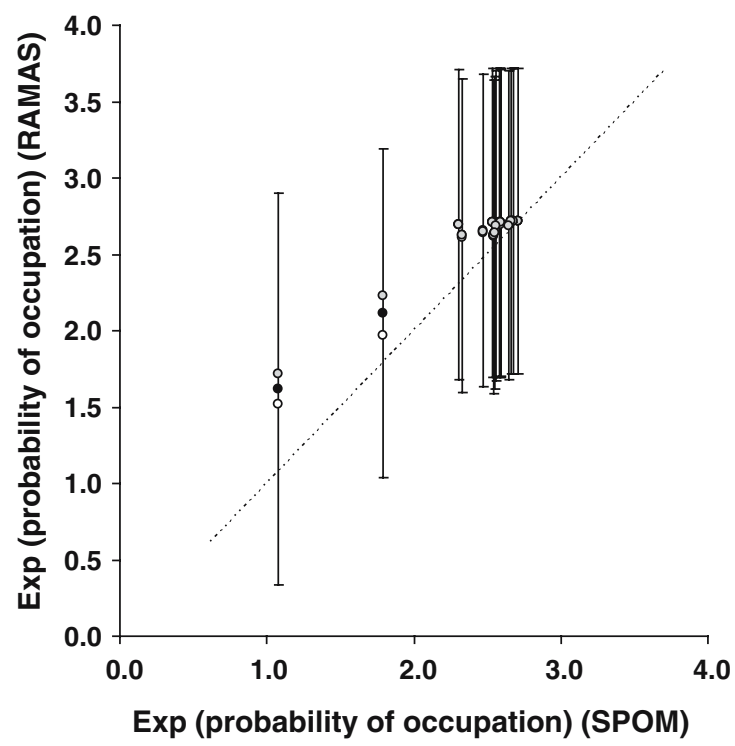

Black, gray and white dots indicate results for the baseline, +10 and $-10 \%$ models, respectively 
Table 1 Sensitivity of the RAMAS model regarding uncertainty in input parameters

\begin{tabular}{llllll}
\hline Parameter & Baseline value & Parameter change $(\%)$ & MPO (SD) (\%) & MFN (SD) & Mean rank \\
\hline Baseline simulation & - & - & $95(6 \%)$ & $217(66)$ & - \\
Maximum growth rate $\left(R_{\max }\right)$ & 2.710 & -50 & $90(9 \%)$ & $130(51)$ & 1.5 \\
Mean dispersal distance $(1 / \lambda)$ & 1.469 & -50 & $88(6 \%)$ & $213(63)$ & 2.5 \\
Dispersal rate $(a)$ & 0.2 & -50 & $93(6 \%)$ & $210(62)$ & 3 \\
SD demographic rates & - & +50 & $94(6 \%)$ & $204(73)$ & 3 \\
Simulation duration & 50 & +50 & $96(5 \%)$ & $217(61)$ & 5 \\
\hline
\end{tabular}

Mean patch occupancy (MPO) and mean final metapopulation size (MFN) are used to rank parameters in a decreasing sensibility order

Table 2 Sensitivity of the stochastic patch occupancy model (SPOM) regarding uncertainty in input parameters

\begin{tabular}{|c|c|c|c|c|c|}
\hline Parameter (abbreviation) & Baseline value & Parameter change $(\%)$ & MPO (\%) & MFN & Mean rank \\
\hline Baseline simulation & - & - & 86 & 312 & - \\
\hline Mean dispersal distance $(1 / \alpha)$ & 1.469 & -50 & 66 & 281 & 1 \\
\hline Colonization $(c)$ & 0.420 & -50 & 78 & 300 & 2 \\
\hline Scaling factor $(\zeta)$ & 0.388 & -50 & 79 & 303 & 3 \\
\hline Extinction $(e)$ & 0.725 & +50 & 81 & 305 & 4 \\
\hline
\end{tabular}

Mean patch occupancy (MPO) and mean final metapopulation size (MFN) are used to rank parameters in a decreasing sensibility order

also appears marginally important, not changing final indicators by more than $6 \%$. Simulation duration appears to have virtually no impact. $\mathrm{A} \pm 10 \%$ modification of the baseline model density-dependence parameters had a limited effect on the outcome of the simulations (Fig. 1).

Sensitivity on the stochastic patch occupancy models

Mean dispersal distance ranked first, again suggesting the importance of dispersal on patch occupancy (Table 2). Species-specific colonization factor $c$ comes second, which again appears logical from a recolonization perspective, while extinction-linked factors ( $e$ and $\zeta$ ) both appear to be of lesser importance, ranking third and last out of four parameters.

\section{Discussion}

Model comparison and sensitivity analyses

The two metapopulation indicators (MPO and MFN) rank populations similarly even though their computation follows very different pathways. The RAMAS model, on one hand, uses local population dynamics (including stage-structured densities) to compute local population sizes and finally metapopulation patterns of occupancy in time. On the other hand, the SPOM uses patch topology and species-specific metapopulationlevel parameters to compute expected equilibrium occupancies. The latter is, therefore, more closely related (both conceptually and in terms of input data) to a metapopulation-level background, while the RAMAS model scales up from the population to the metapopulation level. Nevertheless, there is a very good qualitative concordance between the two approaches in terms of patch ranking. The quantitative discrepancies arise largely from the fact that a patch will be considered occupied in the PSM even if it is occupied by a single individual. It thus indicates that populations in the PSM are frequently unsaturated. In the SPOM, on the other hand, a population will be assumed either occupied with $K_{i}$ individuals or empty.

Absolute predictions of PVA are known to be of limited value, and since both approaches rely on uncertain parameters, sensitivity analysis must be run in both cases in order to interpret their predictions (Taylor 1995; Ruckelshaus et al. 1997; Reed et al. 2002; McCarthy et al. 2003; Lotts et al. 2004) and rank the relative importance of input parameters. Because the two models rely on different input parameters, comparison of sensitivity analyses results is difficult. Nevertheless, it appears that the only parameter they share (mean dispersal distance) ranks as the most and second-most important factor in sensitivity analyses. It thus suggests that the preservation of patch density and landscape permeability is a critical conservation target for the European tree frog. 
Growth rate $\left(R_{\max }\right)$ appears of great importance in the RAMAS sensitivity analysis. Given the large amount of change used $(-50 \%)$, the growth rates slightly exceeded unity on most occasions, which increased the population's likeliness to go extinct from environmental and demographic stochasticity.

Similarly, the SD on demographic rates, which accounts for the major part of environmental stochasticity, appears to be determinant, ranking third out of six parameters.

In conclusion, it appears that although based on fundamentally different paradigms, both modeling approaches are largely concordant in terms of sensitivity to input parameters and mean terminal metapopulation size indices (MPO and MFN). Our results are concordant with Kindvall (2000), who found the incidence function model, logistic regression and RAMAS to be similar regarding turnover rates and regional occupancy. It thus appears that SPOMs provide a lightly parameterized modeling framework that produces results very similar to those of highly parameterized models such as RAMAS, at least in terms of metapopulation persistence indicators and in the ranking of patch values.

In both cases, model parameterization represents the major issue for conservation managers. Data acquisition for both approaches can prove extremely difficult. Depending on the focal species and on data available from previous studies, estimating vital rates (for the PSM) or population turnover rates (for the SPOM) might prove extremely costly and time consuming. Careful attention must be paid to both available data and the ease of additional data acquisition when selecting an appropriate modeling strategy.

\section{Limitations}

There are several aspects of this study that limit the generality of the conclusions that can be drawn from its results. An obvious limitation is that the study considers a single metapopulation of a single species. Similar analyses with other species may result in larger differences between the two types of models studied, especially if the number of populations is changing (e.g., because of habitat fragmentation) or if the populations are declining-either because of declining carrying capacity (e.g., habitat loss or degradation in some habitat patches) or as a density-independent decline (e.g., disease or over-harvest).

Another limitation is that we only considered occupancy and population-size results. A similar analysis of a metapopulation with a substantial risk of extinction may give large differences in extinction risks predicted by the two types of models.
The validation of metapopulation models can usually rely only on the partition of the dataset, the first partition being used for model parameterization and the second for model validation (Kindvall 2000). As our single-species dataset was too small to be partitioned (16 patches), this was an unrealistic option, as is often the case (it is recommended that SPOM be parametrized with larger networks of 30 or more patches, Hanski 1999). Our aim was not to validate the models (which would pose circularity problems given that the parameters were not estimated independently of the patterns observed; see Akçakaya and Sjögren-Gulve 2000; Coulson et al. 2001; Hels and Nachman 2002), but instead to compare their results in terms of ranking of both the values of patches and sensitivity of parameters.

An important limitation of our SPOM is the assumption of equilibrium (stead-state). Many species of conservation concern are declining for various reasons, which invalidates this assumption. In a PSM, such declines are modeled in various ways, e.g., by a gradually declining carrying capacity or by a density-independent decline due to low survival or fecundity. In addition, habitat loss is often accompanied by habitat fragmentation in which the number of patches increases as they split and become smaller. Such changes are incorporated in PSM, but not in SPOM, which severely limits the applicability of SPOM to threatened species.

We assumed in both models that carrying capacities would remain constant (Hanski 2001; Hanski and Ovaskainen 2003; Etienne et al. 2004), which is unlikely to hold since natural succession changes habitat quality. There also is abundant evidence that habitat quality is more important than patch topology in predicting metapopulation dynamics (Thomas et al. 2001; Fleishman et al. 2002; Baguette 2004). Furthermore, the SPOM is assumed to be parameterized from data on steady-state metapopulations. Again, our shortterm metapopulation monitoring (2001-2004) as well as the data provided by Carlson and Edenhamn (2000) are unlikely to fulfill this assumption. Furthermore, our models assume that both demographic rates and landscape structure (patch size and location and dispersing matrix) will remain stable in the future. Because both assumptions are unlikely to hold in an urbanizing landscape (Pellet et al. 2004), the results of our models are weakened (Meir et al. 2004).

\section{Conservation implications}

These metapopulation approaches allow the identification of the critical parameters for the persistence of a European tree frog metapopulation. Both models highlight the importance of dispersal distance. Since it 
is known that $H$. arborea is an excellent disperser (Stumpel and Hanekamp 1986; Edenhamn 1996), it indicates that the actual density of the pond is at the low end of the species' requirements. Although patches are not extremely distant from one another (median=1.54 km; mean=2.17 km; SD=2.06 km), the mean dispersal distance of $H$. arborea $(1.47 \mathrm{~km})$ seems just enough to maintain exchanges between local populations and ensure recolonization. This suggests that patch density is a limiting factor for the European tree frog in our landscape. It is yet another example of the detrimental effects of habitat loss and fragmentation for amphibians (Semlitsch and Bodie 1998; Vos and Chardon 1998; Lehtinen et al. 1999; Marsh and Trenham 2001) to which the European tree frog appears to be extremely sensitive (Edenhamn 1996; Vos et al. 2000). A conservation rule of thumb deduced from our results would be to have a least one suitable pond per $\mathrm{km}^{2}$, thus allowing frogs to maintain sufficient exchanges between patches given their actual mean dispersal distances in agricultural landscapes.

From a modeling perspective, our results also suggest that the solid theoretical framework associated with the SPOM (Hanski and Ovaskainen 2000; Ovaskainen and Hanski 2001) allows a rapid evaluation of metapopulation processes without requiring extensive data on the demography of the focal species. Additionally, their method allows computing the contribution of any new patch to the metapopulation dynamics (Ovaskainen and Hanski 2003). This feature, which is also available within classic PSM such as RAMAS or VORTEX, has a direct conservation application because it allows managers to map the contribution of any new patch in the landscape given its size and location (topology). The combination of such spatially explicit PVA with landscape-scale habitat suitability mapping can certainly help us bridge two fundamental and complementary approaches in threatened species management by identifying the best location for patch creation both from a landscape and a metapopulation perspective.

Acknowledgments This work was funded by the Office Fédéral de l'Environnement, des Forêts et du Paysage, the Center de Conservation de la Faune et de la Nature and the MAVA Foundation. We wish to thank two anonymous reviewers for constructive comments on a previous draft.

\section{References}

Akçakaya HR (2000) Population viability analyses with demographically and spatially structured models. Ecol Bull 48:2338
Akçakaya HR Sjögren-Gulve P (2000) Population viability analysis in conservation planning: an overview. Ecol Bull 48:9-21

Akçakaya HR (2002) Ramas gis: linking landscape data with population viability analysis Version 4.0. Applied Biomathematics Setauket, New York

Akçakaya HR, Burgman MA, Kindvall O, Wood C, SjögrenGulve P, Hatfield J, McCarthy MA (eds) (2004) Species conservation and management: case studies. Oxford University Press, New York

Akcakaya HR (2006) http://www.ramas.com/CMdd.htm\#ddimpact (accessed March 15, 2006)

Alford RA, Richards SJ (1999) Global amphibian declines: a problem in applied ecology. Annu Rev Ecol Syst 30:133-165

Altwegg R (2003) Multistage density dependence in an amphibian. Oecologia 136:46-50

Baguette M (2004) The classical metapopulation theory and the real natural world: a critical appraisal. Basic Appl Ecol 5:213-224

Beissinger SR, McCullough DR (eds) (2002) Population viability analysis. University of Chicago Press, Chicago

Carlson A, Edenhamn P (2000) Extinction dynamics and the regional persistence of a tree frog metapopulation. P Roy Soc Lond B Biol Sci 267:1311-1313

Caswell H (2001) Matrix population models. Sinauer, Sunderland

Conroy SDC, Brook BW (2003) Demographic sensitivity and persistence of the threatened white- and orange-bellied frogs of Western Australia. Popul Ecol 45:105-114

Coulson T, Mace GM, Hudson EJ, Possingham H (2001) The use and abuse of population viability analysis. Trends Ecol Evol 16:219-221

Drechsler M, Frank K, Hanski I, O'Hara R, Wisse C (2003) Ranking metapopulation extinction risk: from patterns in data to conservation management decisions. Ecol Appl 13:990-998

Edenhamn P (1996) Spatial dynamics of the European tree frog (Hyla arborea L.) in a heterogeneous landscape. $\mathrm{PhD}$ Thesis, Swedish University of Agricultural Sciences, Uppsala, Sweden

Etienne RS, ter Braak CJF, Vos CC (2004) Application of stochastic patch occupancy model to real metapopulations. In: Hanski I, Gaggiotti OE (eds) Ecology, genetics and evolution of metapopulations. Elsevier, Burlington, Massachusetts, pp 105-132

Fleishman E, Ray C, Sjögren-Gulve P, Boggs CL, Murphy DD (2002) Assessing the roles of patch quality area and isolation in predicting metapopulation dynamics. Conserv Biol 16:706-716

Friedl TWP, Klump GM (1997) Some aspects of population biology in the European tree frog Hyla arborea. Herpetologica 53:321-330

Grosse WR, Nöllert A (1993) The aquatic habitat of the European tree frog Hyla arborea. In: Stumpel AHP, Tester U (eds) Ecology and conservation of the European tree frog. Institute for Forestry and Nature Research, Wageningen, The Netherlands, pp 37-46

Grossenbacher K (1988) Atlas de distribution des amphibiens de Suisse. Ligue suisse pour la protection de la nature. Basel, Switzerland

Hanski I (1994) A practical model of metapopulation dynamics. J Anim Ecol 63:151-162

Hanski I (1999) Metapopulation ecology. Oxford University Press, Oxford

Hanski I (2001) Spatially realistic theory of metapopulation ecology. Naturwissenschaften 88:372-381 
Hanski I (2004) Metapopulation theory: its use and misuse. Basic Appl Ecol 5:225-229

Hanski I, Ovaskainen O (2000) The metapopulation capacity of a fragmented landscape. Nature 404:755-758

Hanski I, Ovaskainen O (2003) Metapopulation theory for fragmented landscapes. Theor Popul Biol 64:119-127

Hanski I, Simberloff D (1997) The metapopulation approach, its history, conceptual domain and application to conservation. In: Hanski I, Gilpin ME (eds) Metapopulation biology: ecology, genetics and evolution. Academic, London, pp 5-26

Hels T, Nachman G (2002) Simulating viability of a spadefoot toad (Pelobates fuscus) metapopulation in a landscape fragmented by a road. Ecography 25:730-744

Kindvall O (2000) Comparative precision of three spatially realistic simulation models of metapopulation dynamics. Ecol Bull 48:101-110

Lehtinen RM, Galatowitsch SM, Tester JR (1999) Consequences of habitat loss and fragmentation for wetland amphibian assemblages. Wetlands 19:1-12

Lindenmayer DB, Burgman MA, Akçakaya HR, Lacy RC, Possingham HP (1995) A review of three models for metapopulation viability analysis-ALEX RAMAS/Space and VORTEX. Ecol Model 82:161-174

Lopez JE, Pfister CA (2001) Local population dynamics in metapopulation models: Implications for conservation. Conserv Biol 15:1700-1709

Lotts KC, Waite TA, Vucetich JA (2004) Reliability of absolute and relative predictions of population persistence based on time series. Conserv Biol 18:1224-1232

Marsh DM, Trenham PC (2001) Metapopulation dynamics and amphibian conservation. Conserv Biol 15:40-49

Meir E, Andelman S, Possingham HP (2004) Does conservation planning matter in a dynamic and uncertain world? Ecol Lett 7:615-622

Moilanen A, Cabeza M (2002) Single species dynamic siteselection. Ecol Appl 12:913-926

McCarthy MA, Andelman SJ, Possingham HP (2003) Reliability of relative predictions in population viability analysis. Conserv Biol 17:982-989

McCullough DR (1996) Metapopulations and wildlife conservation. Island Press, Washington, DC

Ovaskainen O, Hanski I (2001) Spatially structured metapopulation models: global and local assessment of metapopulation capacity. Theor Popul Biol 60:281-302

Ovaskainen O, Hanski I (2003) How much does an individual fragment contribute to metapopulation dynamics and persistence? Theor Popul Biol 64:481-495

Pellet J, Guisan A, Perrin N (2004) A concentric analysis of the impact of urbanization on the threatened European tree frog (Hyla arborea) in an agricultural landscape. Conserv Biol 18:1599-1606

Pellet J, Hoehn S, Perrin N (2005) Multiscale determinants of tree frog (Hyla arborea L) calling ponds in western Switzerland. Biodivers Conserv 12:2227-2235

Pellet J, Schmidt BR (2005) Monitoring distributions using call surveys: estimating site occupancy detection probabilities and inferring absence. Biol Conserv 123:27-35

Pellet J, Schmidt BR, Fivaz F, Perrin N, Grossenbacher K (2006) Density, climate and varying return points: an analysis of long-term population fluctuations in the threatened European tree frog. Oecologia. DOI 10.1007/s00442-006-0432-1
Reed JM, Mills LS, Dunning JB, Menges ES, McKelvey KS, Frye $\mathrm{R}$, Beissinger S, Anstett M-C, Miller P (2002) Emerging issues in population viability analysis. Conserv Biol 16:7-19

Ruckelshaus M, Hatway C, Kareiva P (1997) Assessing the data requirement of spatially explicit dispersal models. Conserv Biol 11:1298-1306

Schmidt BR, Pellet J (2005) Relative importance of population processes and habitat characteristics in determining site occupancy of two anurans. J Wildlife Manage 69:884-893

Schmidt BR, Zumbach S (2005) Rote Liste der gefährdeten Amphibien der Schweiz. BUWAL-Reihe Vollzug Umwelt, Bern

Schneider H (1993) Behavioural and bioacoustic studies in tree frogs. In: Stumpel AHP, Tester U (eds) Ecology and conservation of the European tree frog. Institute for Forestry and Nature Research, Wageningen, The Netherlands, pp 17-20

Schtickzelle N, Baguette M (2004) Metapopulation viability analysis of the bog fritillary butterfly using RAMAS/GIS. Oikos 104:277-290

Semlitsch RD, Bodie JR (1998) Are small isolated wetlands expendable? Conserv Biol 12:1129-1133

Sjögren-Gulve P (1994) Distribution and extinction patterns within a northern metapopulation of the pool frog Rana lessonae. Ecology 75:1357-1367

Sjögren-Gulve P, Ray C (1996) Using logistic regression to model metapopulation dynamics: large scale forestry extirpates the pool frog. In: McCullough DR (eds) Metapopulations and wildlife conservation. Island Press, Washington, DC, pp 111-137

Sjögren-Gulve P, Hanski I (2000) Metapopulation viability analysis using occupancy models. Ecol Bull 48:53-71

Smith MA, Green DM (2005) Dispersal and the metapopulation paradigm in amphibian ecology and conservation: are all amphibian populations metapopulations? Ecography 28:110-128

Stumpel AHP, Hanekamp G (1986) Habitat and ecology of Hyla arborea in The Netherlands In: Rocek Z (ed) Studies in herpetology. Charles University, Prague, Czech Republic, pp 409-411

Taylor BL (1995) The reliability of using population viability analysis for risk classification of species. Conserv Biol 9:551558

Tester U (1990) Artenschuetzerische relevante Aspekte zur Oekologie des Laubfroschs (Hyla arborea L). PhD Thesis, Medezinische Biologie UNI Basel, Basel, Switzerland

Thomas CD, Bodsworth EJ, Wilson RJ, Simmons AD, Davies ZG, Musche M, Conradt L (2001) Ecological and evolutionary processes at expanding range margins. Nature 411:577-581

Vonesh JR, De la Cruz O (2002) Complex life cycles and density dependence: assessing the contribution of egg mortality to amphibian declines. Oecologia 133:325-333

Vos CC, Chardon JP (1998) Effects of habitat fragmentation and road density on the distribution pattern of the moor frog Rana arvalis. J Appl Ecol 35:44-56

Vos CC, ter Braak CJF, Nieuwenhuizen W (2000) Incidence function modelling and conservation of the tree frog Hyla arborea in The Netherlands. Ecol Bull 48:165-180

Wennergren W, Ruckelshaus M, Kareiva P (1995) The promise and limitations of spatial models in conservation biology. Oikos 74:349-356 